\section{BMJ Paediatrics Open}

\title{
Non-specific aortoarteritis (NSAA) in children: a prospective observational study
}

Himanshu Gupta, ${ }^{1}$ Navjyot Kaur, ${ }^{1}$ Anita Saxena, ${ }^{2}$ Priya Jagia, ${ }^{3}$ Sanjeev Kumar, ${ }^{3}$ Saurabh Kumar Gupta, ${ }^{2}$ Sanjeev Sharma, ${ }^{3}$ Shyam S Kothari, ${ }^{2}$ Sivasubramanian Ramakrishnan (1) ${ }^{2}$

To cite: Gupta $\mathrm{H}$, Kaur N, Saxena A, et al. Non-specific aortoarteritis (NSAA) in children: a prospective observational study. BMJ Paediatrics Open 2021;5:e001106. doi:10.1136/ bmjpo-2021-001106

Received 11 April 2021 Accepted 23 June 2021
Check for updates

(c) Author(s) (or their employer(s)) 2021. Re-use permitted under CC BY-NC. No commercial re-use. See rights and permissions. Published by BMJ

${ }^{1}$ Cardiology, Post Graduate Institute of Medical Education and Research, Chandigarh, India ${ }^{2}$ Cardiology, All India Institute of Medical Sciences, New Delhi, Delhi, India

${ }^{3}$ Cardiac Radiology, All India Institute of Medical Sciences, New Delhi, Delhi, India

Correspondence to Dr Sivasubramanian Ramakrishnan; ramaaiims@ gmail.com

\section{ABSTRACT}

Objective Prospective data on clinical profile, natural history and outcomes of NSAA (non-specific aortoarteritis) in children is limited. We initiated this prospective study to evaluate the short-term and medium-term outcomes of NSAA in children.

Design Prospective observational study.

Setting Tertiary care hospital in India.

Patients We included 28 consecutive children ( $<15$ years) with a diagnosis of NSAA.

Main outcome measures Clinical profile, symptoms, left ventricular ejection fraction (LVEF), the pattern of vessel involvement, complications and markers of disease activity were assessed at the time of diagnosis. All the patients underwent treatment and interventions as per the current guidelines and were followed up for a mean duration of $13.5 \pm 6.7$ months for disease activity and outcomes.

Results The mean age of the cohort was $10 \pm 2.9$ years (14 boys). Twenty (71\%) patients had hypertension. Half of the children presented with acute decompensated heart failure (ADHF). Only 21 patients (75\%) met Sharma et al modified Ishikawa criteria for the diagnosis. The children with active disease (36\%) were managed with immunosuppressive drugs. Percutaneous and surgical interventions were performed in $26(93 \%)$ children. New York Heart Association functional class, LVEF and control of hypertension improved in most children on follow-up. Four children developed vascular restenosis requiring reinterventions. There was no death during mid-term follow-up.

Conclusions Children with NSAA, unlike adults seldom present with classical features of the disease. ADHF and ventricular dysfunction are strikingly common in children. Appropriate immunosuppressive therapy for active disease and timely intervention improves clinical outcomes over a medium term follow-up. Future studies assessing longterm outcome are needed.

\section{INTRODUCTION}

Non-specific aortoarteritis (NSAA) is a granulomatous inflammatory disease of medium to large vessels which results in stenosis, occlusion and less commonly, dilatation of involved arteries. NSAA is also known as Takayasu arteritis after Japanese ophthalmologist, M Takayasu, who described it first in $1908 .^{1}$ The

\section{What is known about the subject?}

Non-specific aortoarteritis (NSAA), though considered a disease of young adults, is the most common cause of renal vascular hypertension in children.

The presentation of NSAA is atypical in children.

\section{What this study adds?}

Children often present with hypertension or acute decompensated heart failure.

- The adult diagnostic criteria for NSAA can miss the disease in as many as $25 \%$ of children.

- Appropriate medical and interventional management results in significant and sustained improvement over a medium term follow-up.

exact aetiology of the disease remains unknown. Though NSAA is primarily considered a disease of young adults, it is being increasingly recognised in children. In fact, it is one of the most common causes of renovascular hypertension in Asian children. ${ }^{2}$ Unlike adults, prospective data on clinical profile, progression and outcomes in children with NSAA are limited. ${ }^{3}{ }^{4}$ In this prospective study, we aimed to study the presentation, course and medium-term outcomes in children with NSAA.

\section{METHODS}

We included all consecutive children (aged $<15$ years) with NSAA after obtaining informed parental consent. The only exclusion criterion was parents' unwillingness to participate in the study. Patients or the public were not involved in the design, or conduct, or reporting, or dissemination plans of our research.

\section{At presentation}

Clinical profile including demographics and presenting symptoms were recorded. Physical examination included assessment of 
peripheral pulses, auscultation for bruits and four limb blood pressure (BP) measurement. The left ventricular ejection fraction (LVEF) was measured using twodimensional echocardiography by two independent experienced operators. We measured markers of renal function and acute phase reactants (APR) including erythrocyte sedimentation rate and $\mathrm{C}$ reactive protein in all the children. Eighteen children suspected to have NSAA directly underwent conventional digital subtraction angiography, while 10 patients were diagnosed based on an initial abdominal ultrasound and Doppler evaluation.

\section{Diagnosis}

Children were diagnosed to have NSAA based on typical angiographic findings in addition to clinical signs and symptoms. Based on the pattern of aortic involvement, five types were identified. ${ }^{56}$

\section{Measurement of activity}

The activity of disease was assessed using US national Institute of Health (NIH) Criteria. ${ }^{7}$

\section{Management and follow-up}

All children diagnosed to have NSAA were managed as per the current guidelines. ${ }^{3}$ Those with evidence of active disease were prescribed immunosuppressive drugs, while those with critical stenotic/valvular lesions were offered appropriate interventions. Angioplasty was considered technically successful if the residual stenosis was $<30 \%$ and the pressure gradient was $<20 \mathrm{~mm} \mathrm{Hg} .{ }^{89}$ All the patients were followed up clinically for evidence of disease activity and development/worsening of new lesions. The LVEF was re-evaluated at 6 months, while imaging and biochemical assessment for NSAA were performed as per the discretion of the treating physician.

\section{Statistical analysis}

Continuous variables are expressed as mean and SD. Categorical values are expressed as frequency and percentages. We applied paired t-test for continuous variables and $\chi^{2}$ test for categorical variables to compare measurements before and after treatment. Mean difference and $95 \% \mathrm{CI}$ are reported as applicable. The difference is considered significant if the $p$ value is less than 0.05. Statistical software used was SPSS Software (V.11.0, SPSS).

\section{Patient and public involvement}

Patients or the public were not involved in the design, or conduct, or reporting, or dissemination plans of our research.

\section{RESULTS}

A total of 28 children (14 boys, $50 \%$ ) were included over 2 years. The mean age at presentation was $10 \pm 2.9$ years (range 6-15years). The most common mode of presentation was acute decompensated heart failure (ADHF) $(\mathrm{n}=14 ; 50 \%)$ and hypertension $(\mathrm{n}=20,71 \%)$ was the most frequent clinical finding. Table 1 summarises the demographic and presenting features of NSAA in our cohort. The BP in the upper and lower limbs was not different ( $p$ value: systolic and diastolic BP difference: 0.22 and 0.38 , respectively). Baseline investigations are summarised in table 2. More than half the children had severe LV dysfunction and New York Heart Association (NYHA) class IV at presentation. The mean NYHA class at presentation was $3 \pm 1.2$.

The classical features of NSAA namely asymmetrical pulses, BP difference between upper limbs (UL) and bruits were found in $4(14 \%), 6(21 \%)$ and $8(29 \%)$ children, respectively. While nine patients $(32 \%)$ had presented with fever and arthralgia, only one patient each had carotidynia and lower limb claudication on presentation. Only 21 patients $(75 \%)$ met Sharma et $a l$ s modified Ishikawa criteria. The mean number of major and minor criteria met by the study population were $1.3 \pm 0.8$ and $2.4 \pm 0.8$, respectively. Fifteen patients (54\%) had dyspnoea on exertion for $>1$ month as major criteria, followed by subclavian artery (SCA) involvement in 10 patients $(36 \%)$. However, all the 28 children could be classified as having NSAA according to the EULAR/ PRINTO/PRES criteria for NSAA. ${ }^{4}$

Descending thoracic aorta (DTA) was the most common part of aorta involved $(\mathrm{n}=13,46 \%)$, and renal artery was the most frequently involved major branch $(\mathrm{n}=12,43 \%)$. Patterns of vessel involvement are represented in figure 1 . The most common type of NSAA in our cohort was type $2(\mathrm{n}=18,64 \%)$, followed by type 3 $(\mathrm{n}=8,29 \%)$. Pulmonary artery involvement (type 4$)$ was seen in only one patient and was clinically silent. Some interesting imaging patterns observed in the cohort are presented in figure 2.

Nineteen patients $(68 \%)$ had $\geq 2$ complications which included LV dysfunction, hypertension, renal failure, aortic regurgitation (AR), retinopathy and aortic aneurysm. LV dysfunction and hypertension were the most common complications, while AR was seen in six patients and abdominal aortic aneurysm and eye involvement in one patient each. Few patients presented with rarer complications of NSAA; one patient had stage-4 retinopathy with bilateral cataract, one patient presented with acute limb ischaemia due to peripheral embolisation from a large LV clot while there was one initial presentation with hypertensive encephalopathy and recurrent seizures. One child had renal dysfunction at presentation.

A total of $10(36 \%)$ patients were diagnosed to have an active disease as per the NIH criteria (raised APR: 9, fever and arthralgia: 9, bruits: 8, arthritis: 2 and carotidynia:1). All the patients with active disease were started on immunosuppressive drugs which included corticosteroids alone or in combination with azathioprine and/or methotrexate.

Interventions were attempted in 26 patients $(93 \%)$ and was successful in 21 patients $(75 \%)$. The patients with active disease were taken up for the procedure 
Table 1 Demographic and clinical characteristics of the study cohort $(n=28)$

SI. no.

Characteristics

Value

Mean \pm SD or N (\%)

(1)

Age in years

$10 \pm 2.9$

Boys

$14(50 \%)$

(3)

Blood pressure

Hypertension

$20(71 \%)$

Highest $\mathrm{BP}$ recorded $\mathrm{UL} \mathrm{mm} \mathrm{Hg}$ (mean $\mathrm{SBP} /$ mean $\mathrm{DBP} \pm \mathrm{SD}$ )

$155.2 \pm 32.6 / 91.9 \pm 21.9$

Highest $\mathrm{BP}$ recorded LL mm Hg (mean SBP/mean DBP \pm SD)

$142.3 \pm 45.4 / 85.5 \pm 28.9$

More than $10 \mathrm{~mm} \mathrm{Hg}$ systolic BP difference between two Uls

(4)

Acute decompensated heart failure

$6(21 \%)$

Presentation in NYHA class IV

$14(50 \%)$

Presentation in NYHA class III

$14(50 \%)$

Presentation in NYHA class II

$4(14 \%)$

Fever and arthralgia

$6(21 \%)$

\begin{tabular}{lll}
\hline$(5)$ & Fever and arthralgia & $9(32 \%)$ \\
\hline$(6)$ & Bruits & $8(29 \%)$ \\
$(7)$ & Unequal pulses & $4(14 \%)$ \\
$(8)$ & Carotidynia & $1(4 \%)$ \\
$(9)$ & Non-cardiac complications of NSAA & $1(4)$ \\
& Retinopathy & $1(4)$ \\
& Encephalopathy and seizures & $1(4)$ \\
& LL claudication & $1(4)$ \\
& Renal failure & $1(4)$ \\
& Abdominal aortic aneurysm & $19(68 \%)$ \\
\hline
\end{tabular}

BP, blood pressure; DBP, diastolic blood pressure; LL, lower limb; LVEF, left ventricular ejection fraction; n, number; NYHA, New York Heart Association; SBP, systolic blood pressure; UL, upper limbs.

usually after remission of activity unless the presentation was ADHF. Successful percutaneous transluminal renal angioplasty (PTRA) was done in six out of nine patients (figure 3A,B), while percutaneous transluminal angioplasty (PTA) was successful in 13 out of 14 attempted procedures (figure 3C,D). PTA was unsuccessful in a

\begin{tabular}{|c|c|}
\hline Variable & $\begin{array}{l}\text { Mean } \pm \text { SD or } \\
\text { N (\%) }\end{array}$ \\
\hline $\mathrm{Hb}(\mathrm{g} / \mathrm{L})$ & $147.9 \pm 16.1$ \\
\hline Platelets (lacs/dL) & $2.1 \pm 0.7$ \\
\hline Urea (mg/dL) & $32.0 \pm 7.4$ \\
\hline Creatinine $(\mathrm{mg} / \mathrm{dL})$ & $0.9 \pm 0.5$ \\
\hline LVEF (\%) & $37.0 \pm 18.3$ \\
\hline LV dysfunction & $19(68 \%)$ \\
\hline Severe LV dysfunction (LVEF $\leq 30 \%$ ) & $15(54 \%)$ \\
\hline Moderate to severe aortic regurgitation & $6(21 \%)$ \\
\hline Mitral regurgitation (more than mild) & $4(14 \%)$ \\
\hline
\end{tabular}

CRP, C reactive protein; ESR, erythrocyte sedimentation rate; $\mathrm{Hb}$, haemoglobin; LVEF, left ventricular ejection fraction. patient with arch involvement where angioplasty to right common carotid artery was attempted. Three patients required stents; two required it for a suboptimal angiographic result after balloon angioplasty and one required it for a large flow-limiting dissection (figure $3 \mathrm{E}-\mathrm{H}$ ). The mean aortic lumen stenosis decreased from $75 \pm 8 \%$ to $23 \pm 10 \% \quad(\mathrm{p}<0.0001)$ and gradient decreased from $60 \pm 15$ to $24 \pm 10 \mathrm{~mm} \mathrm{Hg}(\mathrm{p}<0.0001)$. In the PTRA group, the stenosis decreased from a mean of $90 \pm 7 \%$ to $20 \pm 13 \%$ and the pressure gradient decreased from $70 \pm 27$ to $15 \pm 10 \mathrm{~mm} \mathrm{Hg}(\mathrm{p}<0.001)$. Three patients underwent successful surgery, out of whom two patients required aortic bypass grafting with reimplantation of renal arteries and one patient required mitral valve replacement for severe mitral regurgitation (MR).

Follow-up: Duration of follow-up ranged from 6 to 24 months with a mean of $13.5 \pm 6.7$ months. Twenty-six (93\%) patients were in either NYHA class 1 or 2 on follow-up as compared with 10 patients (36\%) on initial presentation (figure 4). BP was well controlled in 14 $(70 \%)$ patients, with 6 patients $(30 \%)$ requiring only one drug and 3 patients (15\%) requiring none. Mean systolic and diastolic BP decreased significantly as well as the requirement of antihypertensive drugs (table 3). LVEF 


\begin{tabular}{|c|c|c|}
\hline Vessel Involved & $\mathbf{N}$ & $\%$ \\
\hline $\begin{array}{l}\text { Descending Thoradic } \\
\text { Aorta }\end{array}$ & 13 & 46.4 \\
\hline Aortic Arch & 10 & 35.7 \\
\hline Abdominal Aorta & 6 & 21.4 \\
\hline Renal artery & 12 & 428 \\
\hline B/LRA & 5 & 17.8 \\
\hline solated Right RA & 5 & 178 \\
\hline Isolated Left RA & 2 & 7.1 \\
\hline Subdavian artery & 10 & 35.7 \\
\hline B/L SCA & 2 & 7.14 \\
\hline Isolated Right SCA & 3 & 10.71 \\
\hline Isolated Left SCA & 5 & 17.8 \\
\hline Carotid Artery (B/L) & $\mathbf{1}$ & 3.5 \\
\hline
\end{tabular}

Pattern Of Vessels Involvement

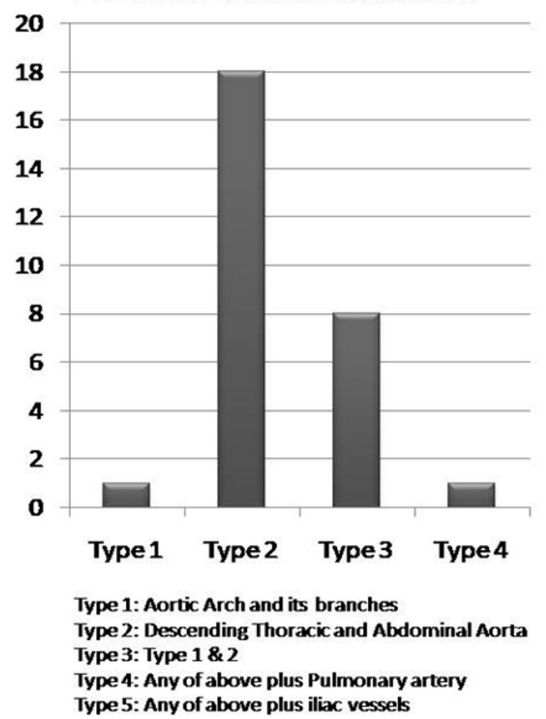

Figure 1 Pattern of vessel involvement observed in the study cohort.

improved in 15 patients $(79 \%)$ with normalisation in 5 patients $(26 \%)$ patients (figure 4$)$.

Out of 21 patients who underwent a successful intervention, 4 patients (19\%) developed restenosis over the follow-up period. Three had to undergo a repeat balloon angioplasty procedure, whereas one patient had to undergo stenting. Three patients had features suggestive of disease activity at follow-up and were started on additional immunosuppressive therapy. All these three patients had activity at the initial presentation. Only two patients $(7 \%)$ had evidence of new vessel involvement. One patient who initially had only arch vessel involvement had significant DTA stenosis at follow-up of 13 months. The second patient who initially had lower DTA and left SCA stenosis developed significant narrowing of suprarenal aorta on follow-up of 12 months. There was no mortality.

\section{DISCUSSION}

Though NSAA is considered predominantly a disease of young adults, ${ }^{10-12}$ it is increasingly recognised in children. $^{3713}$ The data on childhood NSAA have been largely retrospective with older studies focused on the presentation and course of the disease and the recent ones on follow-up of interventions and their outcome. In this study, we present the prospective data of 28 consecutive children with NSAA, their clinical presentation and
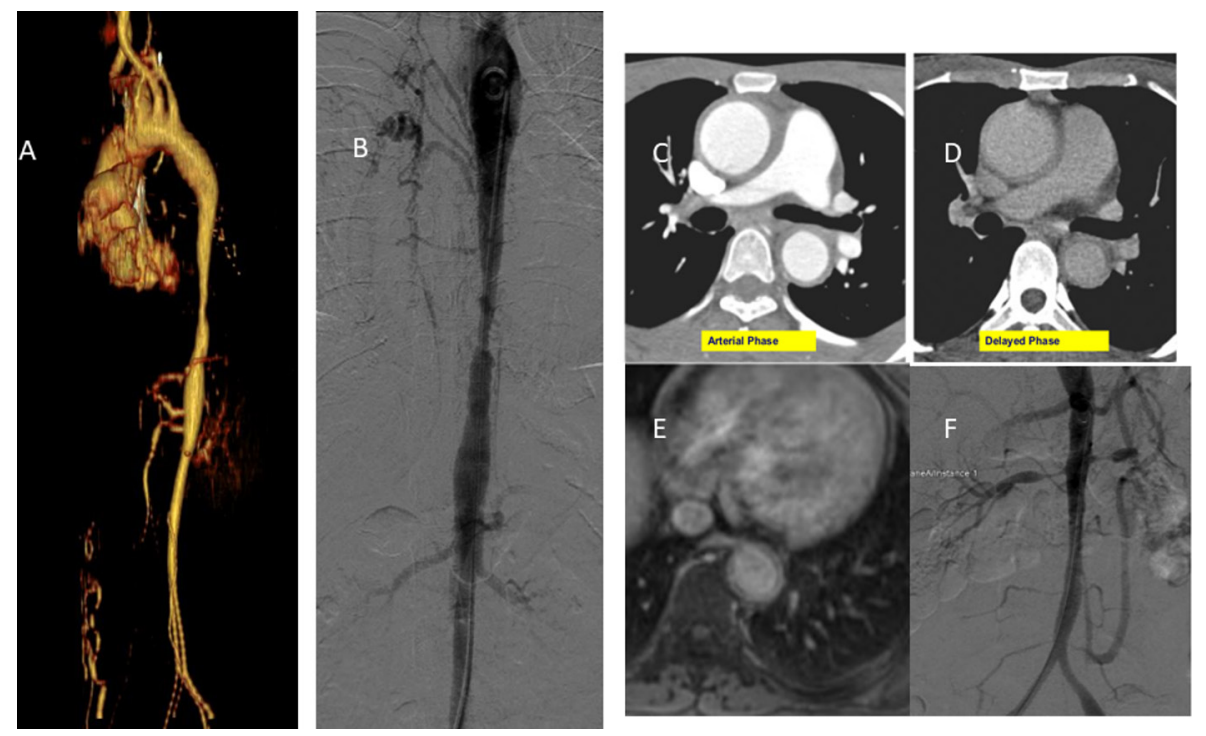

Figure 2 Some selected illustration of imaging patterns observed in the study. (A) A CT-VRT image in sagittal oblique view showing diffuse long segment narrowing of descending thoracic aorta. (B) Corresponding catheter angiogram showing diffuse narrowing with irregular outline. CT angiography arterial (C) and delayed phase (D) images showing wall thickening with enhancement, suggestive of active disease. (E) A MRI showing diffuse wall thickening of thoracic aorta, suggestive of active disease. (F) Catheter angiogram image showing bilateral renal artery stenosis. VRT, volume rendering technique. 

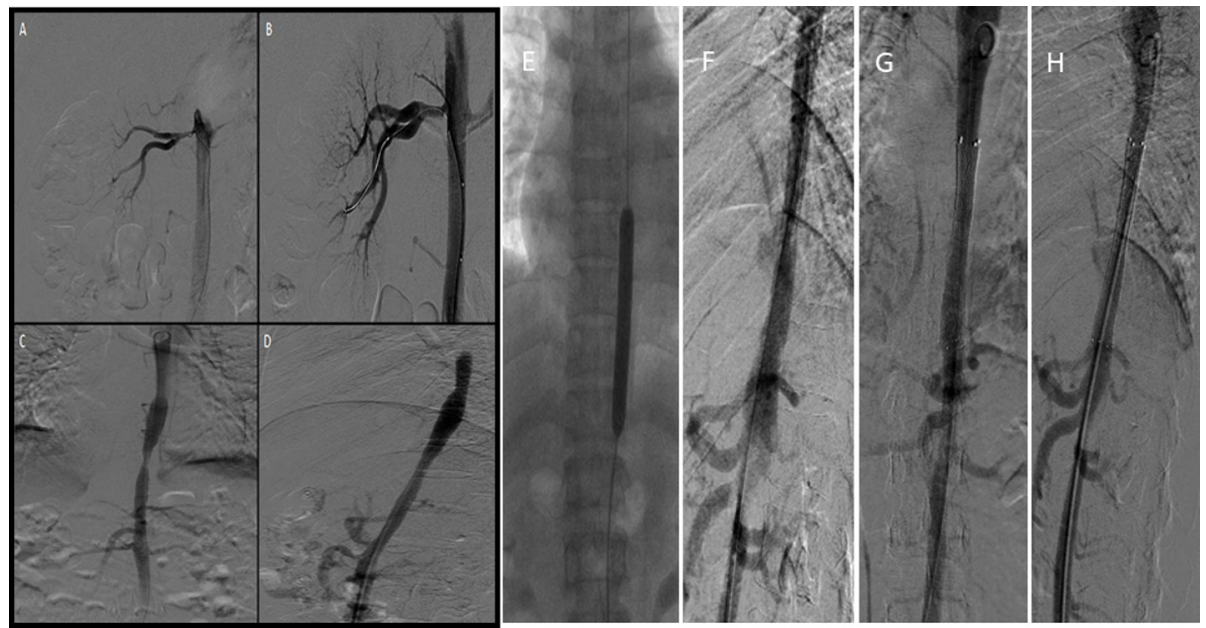

Figure 3 (A) Right renal artery stenosis,. (B) successful PTRA,. (C) aortic stenosis, (D) successful PTA, (E) the lesion was dilated with an appropriate size balloon. (F) However, there was obstructive dissection, which was stented with an appropriate size stent. Check angiogram in anteroposterior $(G)$ and lateral $(H)$ views shows good antegrade flow with no significant stenosis. PRTA, percutaneous transluminal renal angioplasty; PTA, percutaneous transluminal aortoplasty.

mid-term follow-up, with most patients having undergone appropriate interventions.

There have been no validated diagnostic criteria for childhood NSAA. ${ }^{3}$ The Ishikawa diagnostic criteria, ${ }^{14}$ The American College of Rheumatology classification criteria $^{15}$ and Sharma's modification of Ishikawa criteria ${ }^{16}$ used for diagnosis of NSAA in adults, have not been validated for childhood NSAA. ${ }^{3}$ The EULAR/PRINTO/ PRES classification criteria for childhood NSAA, if fulfilled, has a sensitivity and specificity of $99 \%{ }^{4}$ In our cohort, only $75 \%$ of children fulfilled the diagnostic criteria as proposed by Sharma $e t a l$, while all the children could be classified as NSAA as per the EULAR/PRINTO/ PRES classification system.
Unlike in adults, there is no female preponderance of childhood NSAA worldwide. ${ }^{17-19}$ Moreover, childhood NSAA presents less commonly with classical features and more commonly with $\mathrm{ADHF},{ }^{32021}$ as demonstrated in our study also, where $50 \%$ of children had presented with ADHF. Heart failure in NSAA occurs either due to effects of uncontrolled hypertension per se or LV dysfunction secondary to uncontrolled hypertension, AR due to aortic root dilatation, valvular AR or MR, myocarditis or myocardial infarction secondary to coronary involvement. ${ }^{3} 7$ 20-24 Almost $75 \%$ of our patients were found to be hypertensive, which is similar to the previous series on paediatric NSAA. ${ }^{18} 1925$ Hypertension in NSAA is multifactorial and is attributed most commonly to renal

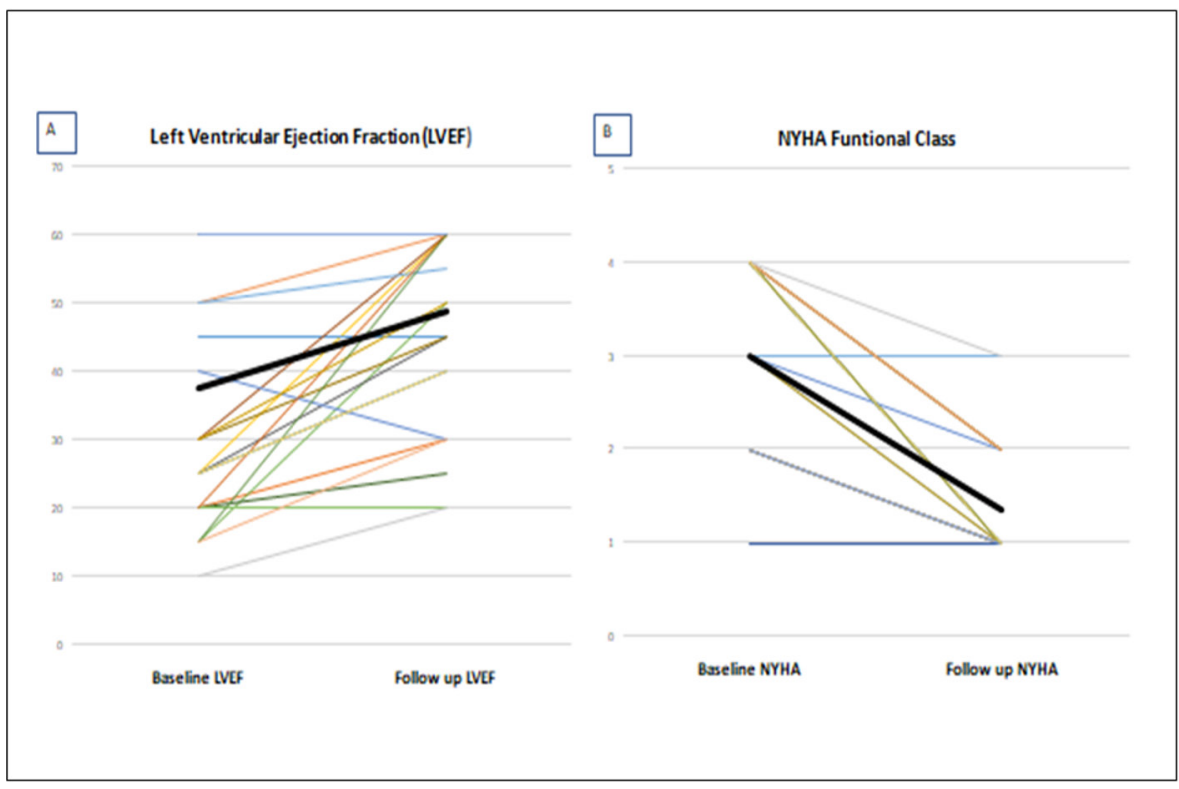

Figure 4 (A) Left ventricular systolic function at baseline and follow-up. NYHA class (dyspnoea) at baseline and follow-up. NYHA, New York Heart Association. 
Table 3 Clinical follow-up of the study cohort $(n=28)$

\begin{tabular}{|c|c|c|c|c|}
\hline $\begin{array}{l}\text { Characteristic } \\
\text { Mean } \pm \text { SD or N (\%) }\end{array}$ & Baseline & Follow-up & Mean difference $(95 \% \mathrm{Cl})$ & $\mathrm{P}$ value \\
\hline Active disease & $10(35.7)$ & $3(10.7)$ & $25 \%(3.9,46.1 \%)$ & 0.02 \\
\hline NYHA functional class & $3.0 \pm 1.2$ & $1.5 \pm 0.7$ & $-1.5(-1.9$ to -1.02$)$ & $<0.001$ \\
\hline LVEF (\%) & $37.0 \pm 18.3$ & $48.4 \pm 13.9$ & $11.4(5.7$ to 17.2$)$ & $<0.001$ \\
\hline \multicolumn{5}{|l|}{ Blood pressure in upper limb } \\
\hline Systolic blood pressure (mm Hg) & $145.8 \pm 32.5$ & $120.4 \pm 22.7$ & $-25.4(-38.0$ to -12.7$)$ & $<0.001$ \\
\hline Diastolic blood pressure (mm Hg) & $87.7 \pm 19.1$ & $77.4 \pm 15.6$ & $-10.3(-18.8$ to -1.8$)$ & 0.02 \\
\hline \multicolumn{5}{|l|}{ Blood pressure in lower limb } \\
\hline Systolic blood pressure (mm Hg) & $128.6 \pm 48.4$ & $119.0 \pm 33.0$ & $-8.8(-8.5$ to 26.1$)$ & 0.31 \\
\hline Diastolic blood pressure $(\mathrm{mm} \mathrm{Hg})$ & $78.1 \pm 29.8$ & $72.3 \pm 17.2$ & $5.7(-5.5$ to 16.7$)$ & 0.30 \\
\hline New vessel involvement at follow-up & - & $2(7.1)$ & - & - \\
\hline
\end{tabular}

LVEF, left ventricular ejection fraction; NYHA, New York Heart Association.

arterial stenosis followed by aortic stenosis and increased aortic and arterial wall stiffness. ${ }^{3} 7922-25$ In fact, NSAA is the most common cause of renovascular hypertension among Asian children. ${ }^{2}$

The classical features of NSAA like constitutional symptoms, unequal pulses, significant BP difference between two arms and carotidynia are less commonly described in childhood NSAA. ${ }^{26} 27$ The reason for this observed difference in the presentation of NSAA in children and adults is that the most frequently involved major branch in childhood NSAA is renal artery rather than SCA as demonstrated in our cohort also. In addition, children often present with BP difference between UL and LL rather than between two ULs, as the descending aorta is the most common part of the aorta involved. The most common pattern of vessel involvement seen in childhood NSAA is type II/III (thoracoabdominal aorta). ${ }^{19} 2829$ In our series, $26(93 \%)$ patients had type II/III disease and $46 \%$ had DTA involvement. There are usually no intercostal collaterals due to the involvement of ostia of these intercostal arteries. Similarly, aneurysms do occur but rarely without stenotic lesions, ${ }^{26} 27$ and in our cohort, only one child had an aneurysm. AR has been reported in $7 \%-15 \%$ patients of $\mathrm{NSAA}^{222430}$ while in our cohort, the incidence was $21 \%$ ( 6 patients). Table 4 summarises the clinical profile and angiographic findings of childhood NSAA in various series. 1928 29 31-34

The most commonly used criteria to define active disease are NIH criteria. ${ }^{5}$ Other scores used are Indian Takayasu Clinical Activity Score ${ }^{35}$ and Disease Extent Index-Takayasu Arteritis consisting of 71 clinical entities. ${ }^{36}$ Clinical activity diagnosed with NIH criteria was seen in 10 of our patients $(36 \%)$ similar to the previous studies, which reported active NSAA in 30\%-50\% cases. ${ }^{19}{ }^{30}$ Most of our patients $(19 ; 68 \%)$ had two or more complications. Severe untreated NSAA has a bad prognosis with a predicted 5-year survival rate of $70 \%$ in patients with $\geq 2$ complications as compared with $100 \%$ in patients with no complications. $^{30}$
All patients with active disease were prescribed immunosuppressive drugs. Studies have shown that $50 \%$ of the patients with NSAA respond to steroids and $50 \%$ of those unresponsive to steroids have remission of disease activity with methotrexate. ${ }^{3} 3738$ Percutaneous intervention was done in 23 children and was successful in 19. While the success rate of PTA $(13 / 14,93 \%)$ was comparable to that described elsewhere in the literature, ${ }^{83839}$ it was definitely lower for PTRA $(6 / 9,67 \%)$ in which few studies have reported a success rate of $95 \% .{ }^{940}$ It may be due to the diffuse nature of the disease in our cohort or possibly is a reflection of small number of children in our study cohort. Like the previous studies, ${ }^{8940}$ we could get excellent control of BP with successful PTA and PTRA. In addition to this, there was also a significant improvement in LVEF, and five patients had normalisation of LVEF on follow-up which is like the previously reported data. ${ }^{8} 2021$

We observed restenosis in $4(19 \%)$ patients. All patients with restenosis had initially presented with disease activity and intervention was done as an emergency measure either because of ADHF or hypertensive emergency. Saxena $e t a l^{8}$ and Tyagi et $a l^{39}$ have reported a restenosis rate of $11.7 \%$ and $19 \%$, respectively, at 6 months of follow-up. Only 2 patients, who had active inflammation at presentation, developed evidence of new vessel involvement on follow-up. There are no data on the incidence of new vessel involvement in children, but a large study in adults ${ }^{30}$ which studied 88 patients and followed them for 83 months had shown that only $15 \%$ of patients show a change in the pattern of peripheral pulses on follow-up. The rare change in vessel involvement at follow-up may indicate a late stage of disease, with no clinical evidence of extension to previously unaffected arteries. Active disease, although, remains a risk factor for new vessel involvement. With reported mortality of $10 \%-30 \%,,^{3} 38$ NSAA remains a serious illness in children. However, there was no mortality in our cohort, which may be due to relatively shorter follow-up. 


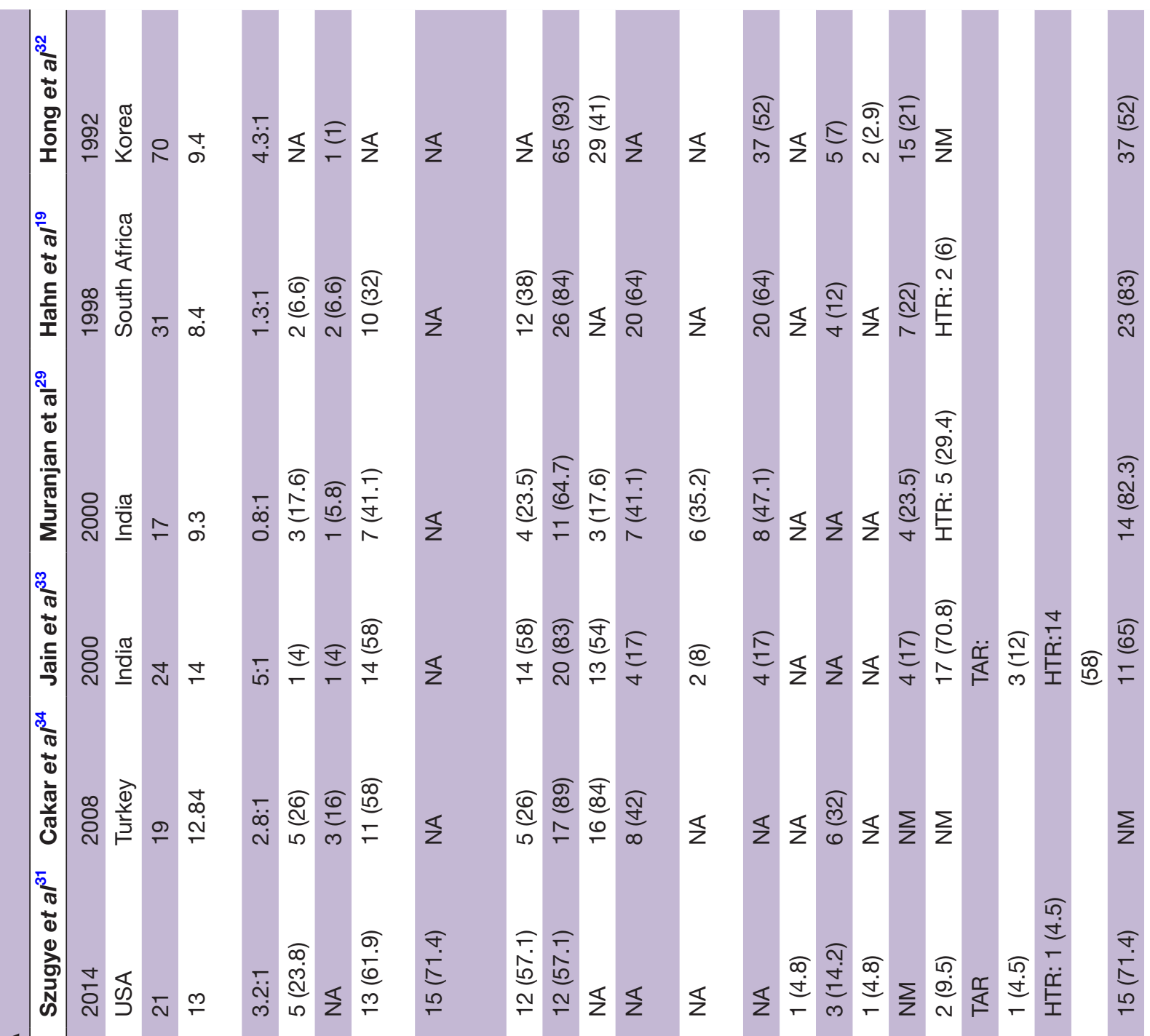

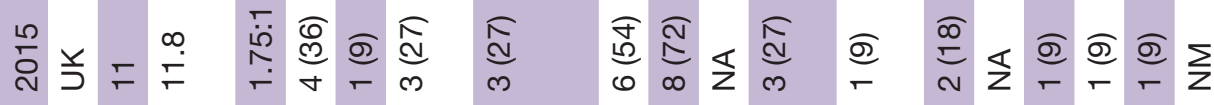

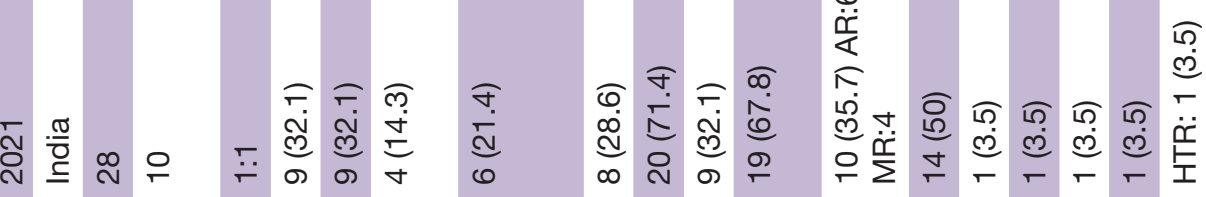

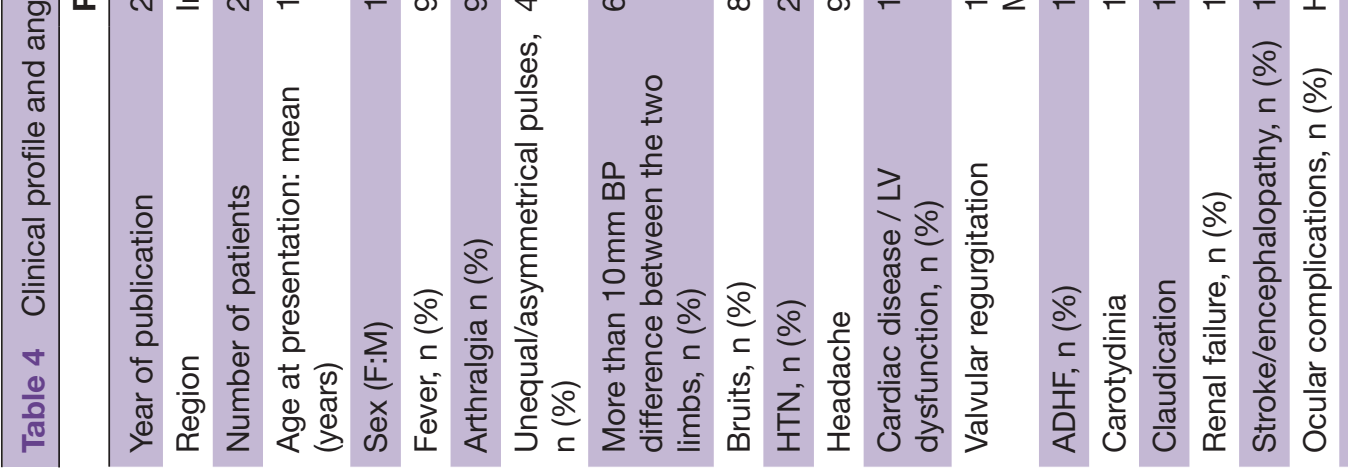




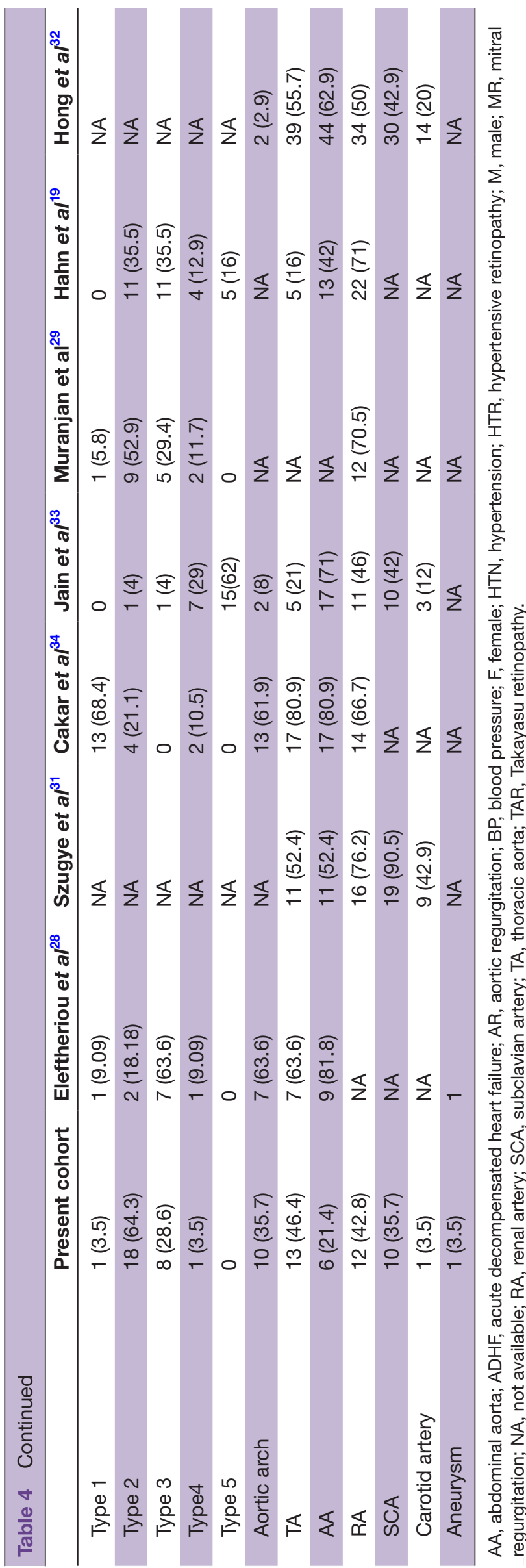

Limitations: The sample size was relatively small and the follow-up of 13.5 months for a chronic inflammatory vasculitis like NSAA is rather short. Since the study was done in a tertiary care setting, children could have been picked up late in the course of disease. The diagnosis was based mostly on typical angiography findings in the presence of clinical suspicion. Follow-up imaging was done only when clinically indicated, which can also underestimate the incidence of new vessel involvement. Childhood NSAA, being an important cause of mortality and long-term morbidity, requires well planned larger and longer prospective studies.

\section{CONCLUSION}

Children with NSAA, unlike adults, seldom present with classical features of the disease, requiring a high index of suspicion for diagnosis. Hypertension remains the most common sign at the time of presentation, and strikingly $50 \%$ of the children present with ADHF. One-third of children have active disease at presentation and almost $70 \%$ of patients had $\geq 2$ complications. However, appropriate immunosuppressive therapy for active disease and timely successful intervention may change the course, with significant improvement in hypertension control, NYHA class and LVEF as illustrated in the prospective medium-term follow-up of the present cohort. Future prospective studies assessing long-term outcomes are needed.

Contributors HG: data collection, writing —original draft preparation. NK: data collection, writing —original draft preparation. AS: planning, conceptualisation, final review and editing. PJ: data collection, literature review and final editing. SK: data collection, literature review and final editing. SKG: data collection, literature review and final editing. SS: planning, conceptualisation, final review and editing. SSK: planning, conceptualisation, final review and editing. SR: planning, conceptualisation, methodology, supervision, final review and editing.

Funding The authors have not declared a specific grant for this research from any funding agency in the public, commercial or not-for-profit sectors.

Competing interests None declared.

Patient consent for publication Not required.

Ethics approval Institute's Ethics committee (AllMS, New Delhi) approved the study vide letter number IEC/NP-288/2011 dated 15 November 2011.

Provenance and peer review Not commissioned; externally peer reviewed.

Data availability statement Data are available on reasonable request. All free text entered below will be published.

Open access This is an open access article distributed in accordance with the Creative Commons Attribution Non Commercial (CC BY-NC 4.0) license, which permits others to distribute, remix, adapt, build upon this work non-commercially, and license their derivative works on different terms, provided the original work is properly cited, appropriate credit is given, any changes made indicated, and the use is non-commercial. See: http://creativecommons.org/licenses/by-nc/4.0/.

ORCID iD

Sivasubramanian Ramakrishnan http://orcid.org/0000-0002-6487-8344

\section{REFERENCES}

1 Takayasu M. Case with unusual changes of the central vessels in the retina (in Japanese). Acta Soc Ophthal Jap 1908;12:554-5. 
2 Chugh KS, Sakhuja V. Takayasu\&rsquo;s arteritis as a cause of renovascular hypertension in Asian countries. Am J Nephrol 1992;12:1-8.

3 Russo RAG, Katsicas MM. Takayasu arteritis. Front Pediatr 2018;6:265.

4 Ozen S, Pistorio A, lusan SM, et al. EULAR/PRINTO/PRES criteria for Henoch-Schönlein purpura, childhood polyarteritis nodosa, childhood Wegener granulomatosis and childhood Takayasu arteritis: Ankara 2008. Part II: final classification criteria. Ann Rheum Dis 2010;69:798-806.

5 Ueno A, Awane Y, Wakabayachi A, et al. Successfully operated obliterative brachiocephalic arteritis (Takayasu) associated with the elongated coarctation. Jpn Heart J 1967;8:538-44.

6 Lupi-Herrera E, Sánchez-Torres G, Marcushamer J, et al. Takayasu's arteritis. clinical study of 107 cases. Am Heart J 1977;93:94-103.

7 Kerr GS, Hallahan CW, Giordano J, et al. Takayasu arteritis. Ann Intern Med 1994;120:919-29.

8 Saxena A, Kothari SS, Sharma S, et al. Percutaneous transluminal angioplasty of the aorta in children with nonspecific aortoarteritis: acute and follow-up results with special emphasis on left ventricular function. Catheter Cardiovasc Interv 2000;49:419-24.

9 Sharma S, Gupta H, Saxena A, et al. Results of renal angioplasty in nonspecific aortoarteritis (Takayasu disease). J Vasc Interv Radiol 1998;9:429-35.

10 Nasu T. Pathology of pulseless disease. A systematic study and critical review of twenty-one autopsy cases reported in Japan. Angiology 1963;14:225-42.

11 Nakao K, Ikeda M, Kimata S, et al. Takayasu's arteritis. clinical report of eighty-four cases and immunological studies of seven cases. Circulation 1967;35:1147-55.

12 Lande A, Bard R, Rossi P, et al. Takayasu's arteritis. A worldwide entity. N Y State J Med 1976;76:1477-82.

13 Ladhani S, Tulloh R, Anderson D. Takayasu disease masquarading as interruption of the aortic arch in a 2-year-old child. Cardiol Young 2001;11:244-6.

14 Ishikawa K. Diagnostic approach and proposed criteria for the clinical diagnosis of Takayasu's arteriopathy. J Am Coll Cardiol 1988;12:964-72.

15 Arend WP, Michel BA, Bloch DA, et al. The American College of rheumatology 1990 criteria for the classification of Takayasu arteritis. Arthritis Rheum 1990;33:1129-34.

16 Sharma BK, Jain S, Suri S, et al. Diagnostic criteria for Takayasu arteritis. Int J Cardiol 1996;54 Suppl:S141-7.

17 Sekiguchi M, Suzuki J. An overview on Takayasu arteritis. Heart Vessels Suppl 1992;7:6-10.

18 Shrivastava S, Srivastava RN, Tandon R. Idiopathic obstructive aortoarteritis in children. Indian Pediatr 1986;23:403-10.

19 Hahn D, Thomson PD, Kala U, et al. A review of Takayasu's arteritis in children in Gauteng, South Africa. Pediatr Nephrol 1998;12:668-75.

20 Varghese MJ, Ramakrishnan S, Salahuddin S. Nonspecific aortoarteritis presenting as acute decompensated heart failure in children: immediate and short-term outcome of percutaneous transluminal renal angioplasty. Ann Pediatr Cardiol 2012;57:103-9.
21 Saxena A, Gupta SK, Kothari S, et al. Aortic obstruction secondary to NSAA in children masquerading as dilated cardiomyopathy. J Am Coll Cardiol 2013;61:E586.

22 Kaul U, Reddy KS, Narula J, et al. Angiographic recognition of coronary ostial stenosis in nonspecific aorto-arteritis. Cathet Cardiovasc Diagn 1988;14:175-9.

23 Chopra P, Singhal V, Nayak NC. Aortoarteritis and cardiomyopathy. A heretofore undescribed association. Jpn Heart J 1978;19:358-65.

24 Talwar KK, Kumar K, Chopra P, et al. Cardiac involvement in nonspecific aortoarteritis (Takayasu's arteritis). Am Heart $J$ 1991;122:1666-70.

25 Panja M, Mondal PC. Current status of aortoarteritis in India. J Assoc Physicians India 2004;52:48-52.

26 Sharma S, Rajani M, Kamalkar T. Clinical and angiographic features of NSAA in children and adults. Acta Radiol 1991;32:485-7.

27 Kothari S. Takayasu's arteritis in children - a review. Images Paediatr Cardiol 2001;3:4-23.

28 Eleftheriou D, Varnier G, Dolezalova P, et al. Takayasu arteritis in childhood: retrospective experience from a tertiary referral centre in the United Kingdom. Arthritis Res Ther 2015;17:36.

29 Muranjan MN, Bavdekar SB, More V, et al. Study of Takayasu's arteritis in children: clinical profile and management. J Postgrad Med 2000;46:3-8.

30 Subramanyan R, Joy J, Balakrishnan KG. Natural history of aortoarteritis (Takayasu's disease). Circulation 1989;80:429-37.

31 Szugye HS, Zeft AS, Spalding SJ. Takayasu arteritis in the pediatric population: a contemporary United States-based single center cohort. Pediatr Rheumatol Online J 2014;12:21.

32 Hong CY, Yun YS, Choi JY, et al. Takayasu arteritis in Korean children: clinical report of seventy cases. Heart Vessels 1992;7:91-6.

33 Jain S, Sharma N, Singh S, et al. Takayasu arteritis in children and young Indians. Int J Cardiol 2000;75 Suppl 1:S153-7.

34 Cakar N, Yalcinkaya F, Duzova A, et al. Takayasu arteritis in children. J Rheumatol 2008;35:913-9.

35 Misra R, Danda D, Rajappa SM, et al. Development and initial validation of the Indian Takayasu clinical activity score (ITAS2010). Rheumatology 2013;52:1795-801.

36 Aydin SZ, Yilmaz N, Akar S, et al. Assessment of disease activity and progression in Takayasu's arteritis with disease extent IndexTakayasu. Rheumatology 2010;49:1889-93.

37 Hoffman GS, Leavitt RY, Kerr GS, et al. Treatment of glucocorticoidresistant or relapsing Takayasu arteritis with methotrexate. Arthritis Rheum 1994;37:578-82.

38 Brunner J, Feldman BM, Tyrrell PN, et al. Takayasu arteritis in children and adolescents. Rheumatology 2010;49:1806-14

39 Tyagi S, Khan AA, Kaul UA, et al. Percutaneous translumina angioplasty for stenosis of the aorta due to aortic arteritis in children. Pediatr Cardiol 1999;20:404-10.

40 Sharma S, Thatai D, Saxena A, et al. Renovascular hypertension resulting from nonspecific aortoarteritis in children: midterm results of percutaneous transluminal renal angioplasty and predictors of restenosis. Am J Roentg 1996;166:157-62. 